
allemande

\title{
Économie de guerre et popularisation des savoirs en Suisse autour de 1917
}

Claire-Lise Debluë

\section{(2) OpenEdition \\ 12 Journals}

Édition électronique

URL : https://journals.openedition.org/allemagne/588

DOI : 10.4000/allemagne.588

ISSN : 2605-7913

Éditeur

Société d'études allemandes

\section{Édition imprimée}

Date de publication : 29 décembre 2017

Pagination : 423-446

ISSN : 0035-0974

\section{Référence électronique}

Claire-Lise Debluë, "Économie de guerre et popularisation des savoirs en Suisse autour de 1917 », Revue d'Allemagne et des pays de langue allemande [En ligne], 49-2 | 2017, mis en ligne le 29 décembre 2018, consulté le 18 mai 2021. URL : http://journals.openedition.org/allemagne/588 ; DOI : https:// doi.org/10.4000/allemagne.588 


\title{
Économie de guerre et popularisation des savoirs en Suisse autour de 1917
}

\author{
- Claire-Lise Debluë*
}

Automne 1917 : alors que le conflit se prolonge, la guerre économique poursuit, dans le domaine des échanges commerciaux, le combat qui fait rage sur les différents fronts. Dans un climat marqué par de fortes tensions sociales, les difficultés d'approvisionnement, le renchérissement et les obstacles aux exportations, la nécessité de justifier l'économie de guerre, d'en expliquer les enjeux et d'en prévenir les effets constitue un défi majeur pour de nombreux États impliqués, directement ou non, dans le conflit. La Première Guerre mondiale marque ainsi un point de rupture incontestable dans la formation et la circulation des savoirs économiques. Si le XIX ${ }^{\text {e }}$ siècle avait été le témoin de l'institutionnalisation et de la professionnalisation de l'économie ${ }^{(1)}$, le court $\mathrm{XX}^{\mathrm{e}}$ siècle qui s'ouvre en 1914 s'affirme comme celui de la popularisation des savoirs économiques. En Suisse, certaines manifestations commerciales directement issues du régime de l'économie de guerre s'imposent comme des armes redoutables dans cette bataille de l'information. À côté de la Foire nationale suisse d'échantillons créée en 1917 à Bâle, la Semaine suisse à laquelle s'intéressera plus particulièrement le présent article, joue un rôle clé dans la popularisation des savoirs économiques. Inaugurée le 27 octobre, la manifestation loue les vertus du patriotisme économique et met le «travail national» à l'honneur. Détaillants et commerçants proscrivent une semaine durant les marchandises importées de leurs devantures. Cette année-là, les 20000 vitrines réparties sur l'ensemble du territoire national, décorées aux couleurs de la manifestation, ont pour mission d'élever «la conscience intérieure du peuple»

* Chercheur post-doc Fonds national suisse de la recherche, Centre des sciences historiques de la culture, Université de Lausanne.

1 Voir Massimo M. Augello, Marco E.L. Guidi, «Nineteenth-Century Economic Societies in a Comparative Approach: the Emergence of Professional Economists», in: Massimo M. Augello, Marco E. L. Guid, The Spread of Political Economy and the Professionalisation of Economists, London/New York, Routledge, 2007, p. 1-32. 
en l'aidant à former une «juste appréciation des capacités économiques de notre petit pays " ${ }^{(2)}$. Recrutés parmi les milieux patriotes de la Nouvelle droite, les organisateurs de la Semaine suisse poursuivent un double objectif: favoriser l'écoulement des "produits indigènes " ${ }^{(3)}$ sur le marché intérieur et diffuser, auprès d'un large public généralement peu familiarisé avec les enjeux de la politique commerciale, les rudiments de la «lutte contre la concurrence étrangère» (wirtschaftliche Überfremdung) - ce nouvel axiome de l'économie de guerre. Dès 1917, les organisateurs de la Semaine suisse contribuent ainsi à faire émerger dans la sphère publique un discours de "persuasion économique» ${ }^{(4)}$ qui fournit un modèle explicatif aux grandes mutations structurelles entraînées par l'éclatement de la guerre.

Si, depuis les années 1990, de très nombreux travaux ont été consacrés aux conditions de formation des savoirs scientifiques et à leur circulation dans la culture visuelle ${ }^{(5)}$, les chemins empruntés pour diffuser auprès du grand public les savoirs économiques ont rarement fait l'objet d'une analyse systématique ${ }^{(6)}$. Ce sont essentiellement les conditions qui présidèrent à la formulation d'un langage visuel de la statistique économique et sociale, selon une approche le plus souvent didactique (du Gesellschafts- und Wirtschaftsmuseum à Vienne aux campagnes de propagande menées dans le cadre du New Deal), qui ont été analysées ${ }^{(7)}$. Le phénomène demeure ainsi largement méconnu pour la période de la Première Guerre mondiale. Pourtant, comme l'ont montré Christophe Bonneuil et Dominique Pestre, «le siècle qui commence avec la Première Guerre mondiale est celui de la pénétration des savoirs scientifiques et de leurs imaginaires dans tous les aspects de la vie sociale, économique et culturelle» ${ }^{(8)}$. Ce changement majeur qui se traduit notamment par une «multiplication des espaces de savoir et des conflits

2 «Semaine suisse. Soleure, le 9 octobre 1917, À Monsieur le Directeur de l'Instruction publique du Canton de __, Schweizerisches Wirtschaftsarchiv (CH SWA), Verbandsarchiv Armbrust/Schweizer Woche, PA 486, B 72.

3 Semaine suisse, Lausanne, Imprimerie vaudoise, s. d. (c. $1^{\text {er }}$ août 1917), p. 1, CH SWA, Verbandsarchiv Armbrust/Schweizer Woche, PA 486, B 72.

4 Marion Fourcade, Economists and Societies. Discipline and Profession in the United States, Britain and France, 1890s to 1990s, Princeton/Oxford, Princeton University Press, 2009, p. 175 sq.

5 Voir par exemple le numéro spécial de la revue Early Popular Visual Culture, consacré au thème «Victorian Science and Popular Visual Culture», 10/1 (février 2012).

6 Voir notamment Simon Cook, «Late Victorian Visual Reasoning and Alfred Marshall's Economic Science», The British Journal for the History of Science, 38/2 (juin 2005), p. 179-195; Anders Екsтröм, "'Showing at One View': Ferdinand Boberg's 'Statistical Machinery' and the Visionary Pedagogy of Early Twentieth-Century Statistical Display», Early Popular Visual Culture, 6/1 (2008), p. 35-49.

7 Sur Otto Neurath et le Gesellschafts- und Wirtschaftsmuseum, voir par exemple: Frank Hartmann, Erwin K. BAUER, Bildersprache. Otto Neurath Visualisierung, Vienne, WUV Universitätsverlag, 2002; Nader Vossoughian, Otto Neurath. The Language of the Global Polis, Rotterdam, NAi Publishers, 2008. Sur la diffusion de la Bildstatistik aux États-Unis, dans le cadre des campagnes du New Deal, voir Loïc Charles, Yann Giraud, «Economics for the Masses: The Visual Display of Economic Knowledge in the United States (1921-1945)», Thema (Théorie économique, modélisation et applications), $\mathrm{n}^{\circ} 3$, Working paper (juin 2010), p. 1-46. Signalons également le numéro thématique de Genèses, paru en 2013, consacré à la "pédagogie économique» après 1945, même si cette question n'est pas traitée du point de vue de l'histoire visuelle.

8 Christophe Bonneuil, Dominique Pestre, «Le siècle des technosciences (depuis 1914)», in: Dominique Pestre (dir.), Histoire des sciences et des savoirs, Paris, Éditions du Seuil, 2015, vol. 3, p. 9. 
autour de ce qui constitue un savoir légitime» ${ }^{(9)}$, n'est pas propre au seul champ des sciences naturelles ou physiques. Il concerne également celui de l'économie.

En resituant la Semaine suisse dans une dynamique transnationale, cet article s'interrogera sur les types de savoirs mobilisés dans le cadre de l'économie de guerre, sur leurs usages et sur leurs aires de circulation. Dans un premier temps, nous montrerons que l'essor de nouvelles formes de visualisation de l'information de l'économie s'inscrit dans un mouvement plus large de professionnalisation et d'institutionnalisation des savoirs économiques. Si ce phénomène se traduit de manière particulièrement manifeste à la fin du XIX ${ }^{\mathrm{e}}$ siècle dans le domaine de la statistique graphique, la création d'expositions, de musées et de collections de guerre, destinés à un public nonspécialiste, favorise dès 1914 le décloisonnement des savoirs économiques. Envisagée dans cette double généalogie, la Semaine suisse n'apparaît plus seulement comme une manifestation issue de l'économie de guerre, mais également comme un agent clé de la reconfiguration des savoirs. C'est à la démonstration de cette hypothèse que la deuxième partie de notre article sera consacrée. Nous montrerons ainsi que, mus par la volonté «d'exercer une influence éducatrice sur tout le peuple ${ }^{(10)}$, les organisateurs de la Semaine suisse ont cherché à mettre en place un enseignement de type empirique, dont la vitrine deviendra sinon l'unique instrument du moins l'emblème privilégié.

\section{L'essor de l'iconographie économique au tournant du $\mathrm{XX}^{\mathrm{e}}$ siècle: de l'institutionnalisation à la vulgarisation des savoirs}

Contrairement aux sciences de la nature qui, dans les sociétés libérales de la deuxième moitié du XIX ${ }^{\mathrm{e}}$ siècle, avaient été au cœur d'un vaste mouvement de popularisation des savoirs ${ }^{(1)}$, l'économie n'avait guère connu un tel engouement. Si l'essor de la presse illustrée, de la photographie, puis du cinéma avait contribué à construire les savoirs scientifiques comme un objet de communication de masse, l'économie était considérée, au début du $\mathrm{XX}^{\mathrm{e}}$ siècle encore, comme une discipline jouissant d'une faible légitimité au sein du champ des sciences sociales ${ }^{(12)}$. Longtemps réservée à un cénacle d'experts, l'économie s'impose peu à peu dans le débat politique et public. L'introduction d'enseignements disciplinaires dans les universités, la création d'instituts de recherche et le lancement de publications spécialisées transforment progressivement le regard porté sur cette discipline. Alors même qu'elle parachève son processus de professionnalisation et d'institutionnalisation, de nouvelles formes de vulgarisation des savoirs économiques voient en effet le jour. Le phénomène est particulièrement frappant dans le domaine de la statistique, où les vertus épistémiques de la statistique dite graphique - par opposition à la statistique «numérique» - sont largement débattues au sein des différentes sociétés savantes. Réputée pour ses vertus interprétatives,

9 Ibid., p. 15.

10 Semaine suisse (note 3), p. 12.

11 Dave Muddiman, «Science, Industry and the State: Scientific and Technical Information in EarlyTwentieth-Century Britain", in: Alistair Black, Dave Muddiman, Helen Plant (éd.), The Early Information Society. Information Management in Britain before the Computer, Aldershot, Ashgate, 2007, p. 56.

12 Timothy Shenk, Timothy Mitchell, «Les savoirs de l'économie», in: D. Pestre (dir.), Histoire des sciences et des savoirs (note 8), p. 234. 
ses qualités « objectives » et sa capacité à frapper l'imagination, la statistique graphique est conçue comme une véritable "langue scientifique» ${ }^{(13)}$. "Bientôt la statistique ne pourra pas plus se passer de gravures que ne s'en passent la géographie, l'anatomie, la mécanique, etc. » ${ }^{(14)}$, présage ainsi en 1895 le statisticien français Jacques Bertillon. C’est à travers certains supports illustrés extrêmement répandus dans le champ scientifique, comme les atlas ou les planches encyclopédiques, que les images économiques commencent, elles aussi, à circuler dès la fin du XIX $\mathrm{X}^{\mathrm{e}}$ siècle.

Lors de l'Exposition nationale suisse de 1896 à Genève, un grand nombre de "tableaux graphiques coloriés» ${ }^{(15)}$ sont notamment présentés au public. Les planches illustrées, imprimées en couleurs, sont réunies dans un Atlas graphique et statistique qui paraît l'année suivante. De facture extrêmement soignée, les illustrations reproduites se distinguent des longues colonnes de chiffres ordinairement publiées dans les ouvrages spécialisés (fig. 1). «N’est-ce pas là une preuve

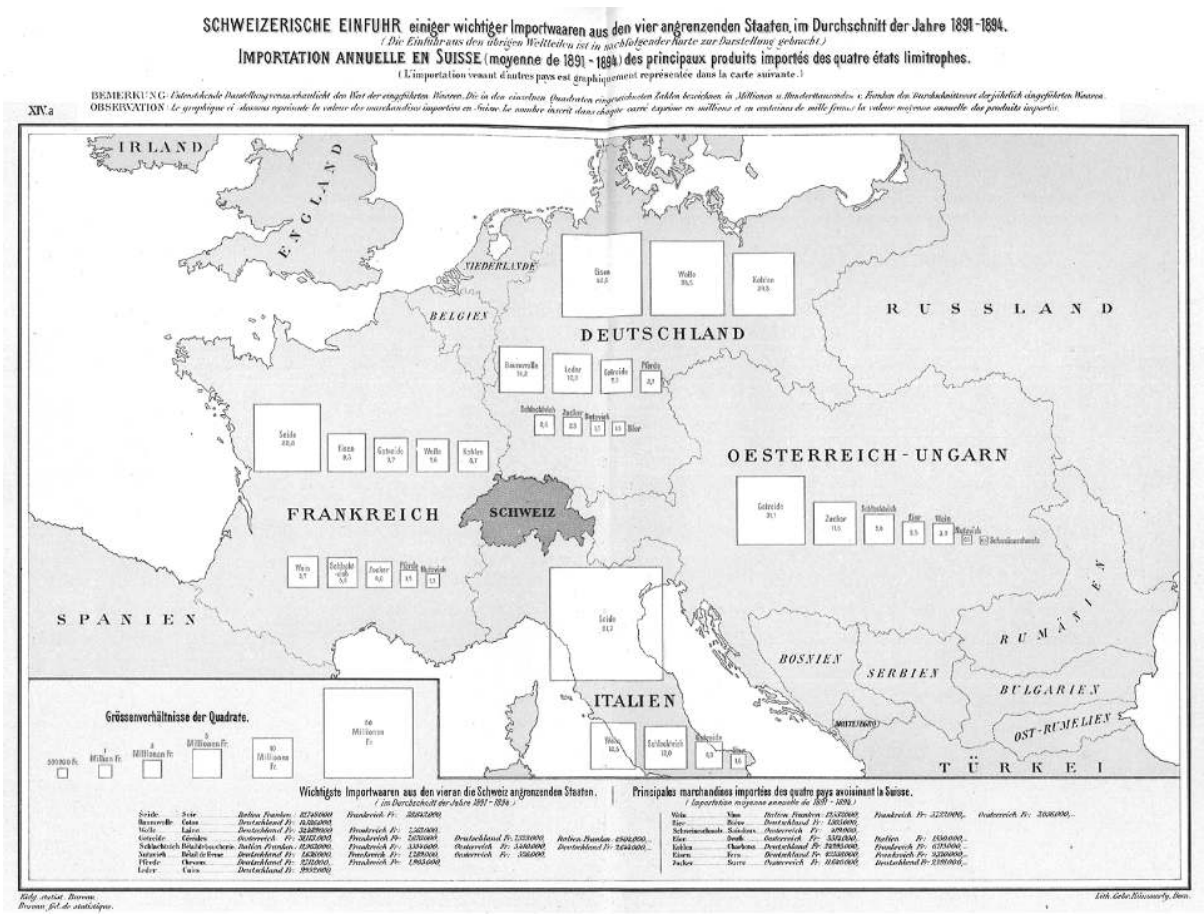

Fig. 1 : «Importation annuelle en Suisse»

Paru dans Atlas graphique et statistique de la Suisse, publié par le Bureau statistique du Département fédéral de l’Intérieur, Berne, Burchdruckerei Stämpfli \& Cie, 1897, (s.p.)

13 Émile Levasseur, «La statistique graphique», Journal of the Statistical Society of London, 22-24 juin 1885, p. 248, en ligne: www.jstor.org/stable/25163975 (consulté le 25.04.2016).

14 Jacques Bertillon, Cours élémentaire de statistique administrative, Paris, Société d'éditions scientifiques, 1895, p. 122.

15 «Avant-propos», in: Atlas graphique et statistique de la Suisse, publié par le Bureau statistique du Département fédéral de l’Intérieur, Berne, Burchdruckerei Stämpfli \& Cie, 1897, p. III. 
que la statistique n'est plus considérée de haut comme c'était encore le cas, il y a quelques années, par une grande partie de la population?", s'interroge-t-on dans l'avant-propos de l'ouvrage comme pour montrer la légitimité gagnée par ce type de visualisation de l'information économique ${ }^{(16)}$. En Suisse, les expositions nationales jouent, il est vrai, un rôle important dans la circulation des savoirs industriels, scientifiques ou artistiques. Consacrées comme des lieux voués à l'édification et au divertissement des masses, elles se prêtent, à plus forte raison encore, à devenir le laboratoire de nouvelles pratiques visuelles de l'économie. À l'instar de la cartographie, devenue une discipline extrêmement populaire dans les expositions de la fin du $\mathrm{XIX}^{\mathrm{e}}$ siècle, la statistique, appréciée pour sa capacité d'abstraction et sa prétendue transparence, contribue à la construction des identités nationales. En tant que méthode quantitative, elle façonne autant qu'elle objective le concept d' "économie politique " ${ }^{(17)}$. La statistique participe enfin à en établir certains des principes dans la culture visuelle, sous formes de diagrammes, de courbes ou de cartogrammes. L'engouement bien réel suscité par ces nouvelles formes de visualisation de l'information économique se heurte toutefois à la méfiance et à la résistance de certains acteurs du champ statistique redoutant que la «popularisation» de la discipline ne nuise à l'entreprise de légitimation amorcée par ses pionniers depuis la fin du XIX ${ }^{\mathrm{e}}$ siècle $^{(18)}$. Au tournant du siècle en effet, d'autres formes de visualisation, prétendant concilier l'objectivité scientifique et l'accès à la connaissance, émergent progressivement et contribuent à diversifier les usages de la statistique graphique. Les effets de la Grande crise et l'avènement de la «question sociale» conduisent notamment le mouvement dit réformiste à s'emparer de la statistique pour dénoncer, chiffres à l'appui, les inégalités persistantes ${ }^{(19)}$. Au-delà des expositions nationales ou universelles ${ }^{(20)}$, dans les centres de documentation, les bibliothèques et les musées sociaux créés en Europe et aux États-Unis entre la fin du XIX ${ }^{\mathrm{e}}$ siècle et le début du $\mathrm{XX}^{\mathrm{e}}$ siècle, l'économie devient un objet divertissant de connaissance, comme l'avaient été avant elle les sciences naturelles et physiques ${ }^{(21)}$. On parle alors volontiers de statistique "populaire» ou même "artistique» ${ }^{(22)}$. Simples objets de contemplation ou d'étude, fidèles à la tradition des atlas scientifiques, les tableaux statistiques font place à de véritables machines, qui permettent au visiteur de s'approprier - parfois littéralement - la

16 Ibid.

17 J. Adam Tooze, «Imagining National Economies: National and International Economic Statistics, 1900-1950 », in: Geoffrey Cuвiтt (éd.), Imagining Nations, Manchester/New York, Manchester University Press, 1998, p. 213.

18 Hans Schorer, «Die graphische Statistik an der schweizerischen Landesausstellung Bern 1914», Journal de statistique suisse, 4/50 (1914), p. 350.

19 Jakob TAnner, «Der Tatsachenblick auf die "reale Wirklichkeit” : zur Entwicklung der Sozial- und Konsumstatistik in der Schweiz», Revue suisse d'histoire, vol. 45 (1995), p. 95.

20 А. Екsтröм, «'Showing at One View' (note 6), p. 35-50.

21 Bernard Lightman parle de «valeur de divertissement de la connaissance» («entertainment value of knowledge»), in: Bernard Lightman, «Victorian Science and Popular Visual Culture», Early Popular Visual Culture, 10/1 (février 2012), p. 1.

22 Heinrich Schlosser, «Künstlerische statistische Darstellungen », (Das) Werk, 2/1 (1915), p. 8. 
matière exposée (fig. 2). De manière générale, le développement des usages de la statistique graphique au tournant du $\mathrm{XX}^{\mathrm{e}}$ siècle témoigne de l'existence d'une sensibilité nouvelle aux vertus éducatives de l'iconographie économique. La multiplication des aménagements tridimensionnels, privilégiés pour leur matérialité et leur potentiel d'interactivité, s'inscrit pleinement dans les changements intervenus à la même période dans l'enseignement d'autres disciplines, comme l'histoire locale, qui recourent de plus en plus volontiers à des systèmes de documentation librement accessibles dans les bibliothèques publiques ${ }^{(23)}$.

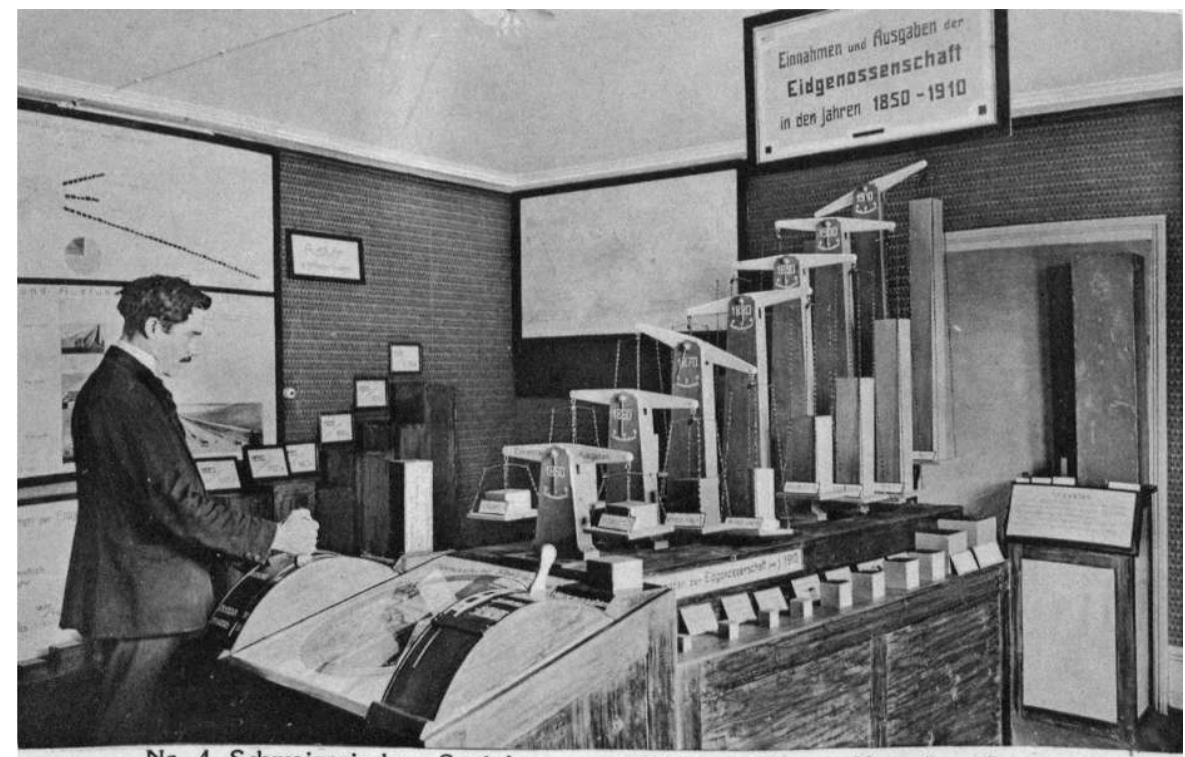

Nr. 4 Schweizerisches Sozialmuseum Zürich - Raum "Vermögen“

Fig. 2: Appareil exposé au Musée social suisse de Zurich (Schweizerisches Sozialmuseum), présentant, au propre comme au figuré, la balance commerciale de la Suisse Carte postale, Schweizerisches Sozialarchiv, 000/37 Z 1

\section{Exposer l'économie de guerre: de la collecte d'information à la visualisation}

L'émergence de nouvelles pratiques de l'exposition de l'économie est fortement favorisée par un autre phénomène : la création de collections et de musées de guerre ${ }^{(24)}$. En Allemagne, en Autriche, en Grande-Bretagne ou en France, de très nombreux centres d'archives, collections et musées consacrés à la guerre sont en effet inaugurés. Rien que dans les Empires centraux, 450 «collections de guerre» voient le jour entre 1914 et $1920^{(25)}$. Statistiques, imprimés, cartes postales, photographies, mais

23 Elizabeth Edwards, «Photographs, Mounts, and the Tactile Archive», Interdisciplinary Studies in the Long Nineteenth Century, ${ }^{\circ} 19$ (2014), en ligne: http://doi.org/10.16995/ntn.716

24 Thomas Thiemeyer, Fortsetzung des Krieges mit anderen Mitteln. Die beiden Weltkriege im Museum, Paderborn, Ferdinand Schöningh, 2010, p. 70.

25 Aibe-Marlene Gerdes, «Kriegssammlungen 1914-1918. Eine Einführung. Die Spuren des Krieges sammeln", in: Julia Freifrau Hiller von Gaertringen (éd.), Kriegssammlungen 1914-1918, Francfort, Vittorio Klostermann, 2014, p. 15. 
aussi certains objets jugés emblématiques, y sont généralement conservés dans le but de documenter la production éditoriale de la période de guerre. D’un cas à l'autre, les missions de ces différentes institutions varient considérablement. À l'Université de Iéna par exemple, où des «archives de guerre» (Kriegsarchiv) sont créées en 1917, la collection est tenue à la disposition des usagers de la bibliothèque qui souhaitent s'en servir pour leurs recherches ou leurs enseignements ${ }^{(26)}$. D'autres institutions mettent davantage l'accent sur l'ouverture de leurs collections au public. Renouant avec la tradition des musées industriels créés au XIX ${ }^{\mathrm{e}}$ siècle à l'issue des révolutions libérales, certains musées de guerre deviennent ainsi de véritables lieux de formation économique. L'exemple le plus frappant est sans doute celui de Leipzig où un "Musée allemand de l'économie de guerre»(Deutsches Kriegswirtschaftsmuseum) est inauguré en mai 1918 et dont la direction est confiée à Otto Neurath, ce pionnier de la Bildstatistik, qui se fera connaître dans le monde entier à la fin des années 1920 grâce à ses travaux réalisés dans le cadre du Gesellschafts- und Wirtschaftsmuseum de Vienne. Dédié à un public spécialiste formé au sein des écoles commerciales et des universités, aussi bien que profane, le Musée a ainsi pour vocation de vulgariser les savoirs de l'économie de guerre à travers «des mots et des images » ${ }^{(27)}$.

Si les collections et les musées de guerre contribuent à façonner de nouveaux savoirs - en matière économique notamment -, les «expositions d'images de guerre» (Kriegsbilderausstellungen) participent également à la diversification des usages pédagogiques de l'image. Les «images de guerre» (Kriegsbilder) - qu'il s'agisse de dessins, de photographies ou, plus rarement, de peintures réalisées sur le front - sont particulièrement appréciées pour leur capacité à «perpétuer et animer le souvenir» ${ }^{(28)} \mathrm{du}$ conflit. Dans une «langue compréhensible par tous " ${ }^{(29)}$ qu'une multitude de mots ne saurait, estime-t-on, exprimer avec une telle clarté, les artistes fidèles à la tradition de l'iconographie militaire se prêtent ainsi régulièrement à l'exercice de la propagande patriotique (fig. 3). La force suggestive attribuée aux images et, sur un plan plus pratique, la facilité avec laquelle celles-ci peuvent être mises en circulation, contribuent à l'autonomisation des «expositions d'images de guerre» en tant que genre. Itinérantes, ces expositions font halte dans différentes villes de chacun des deux camps belligérants. Dès 1916, de nombreuses galeries et académies d'art des Empires centraux et de

26 «Satzung des Kriegsarchivs der Universitätbibliothek zu Jena» (s. d., c. 20 janvier 1917), Kriegsarchiv der Universitätsbibliothek Jena, 75-421, en ligne: archive.thulb.uni-jena.de/hisbest/rsc/viewer/ HisBest_derivate_00002086/Delbrueck_075_0421.tif (consulté le 02.05.2016).

27 Sur le Deutsches Kriegswirtschaftsmuseum, voir Otto Neurath, «Die Kriegswirtschaftslehre und ihre Bedeutung für die Zukunft», Vortrag, gehalten im Städtischen Kaufhaus in Leipzig am 4. Mai 1918, Veröffentlichungen des Deutschen Kriegswirtschaftsmuseums zu Leipzig, $\mathrm{n}^{\circ}$ 4, Leipzig, 1918, publié dans Rudolf Haller, Ulf Töfer (éd.), Otto Neurath. Gesammelte ökonomische, soziologische und sozialpolitische Schriften (II), Vienne, Verlag Hölder-Pichler-Tempsky, vol. 5, p. 596. Voir également Günther SAndner, Otto Neurath. Eine politische Biographie, Vienne, Paul Zsolnay Verlag, 2014, p. 85-86 et Nader Vossoughian, "The War Economy and the War Museum: Otto Neurath and the Museum of War Economy in Leipzig, c. 1918", in: Elisabeth Nemeth, Stefan W. Schmitz, Thomas Uebel (éd.), Otto Neurath's Economics in Context, Vienne, Springer, 2007, p. 131-139.

28 Je traduis. Max Ritter von Hoen, «Kunst», in: Offizieller Katalog der Kriegsausstellung. Wien, 1916, Mai-Oktober K. K. Prater Kaisergarten, Vienne, Industrie, 1916 (3éd.), p. 172. 


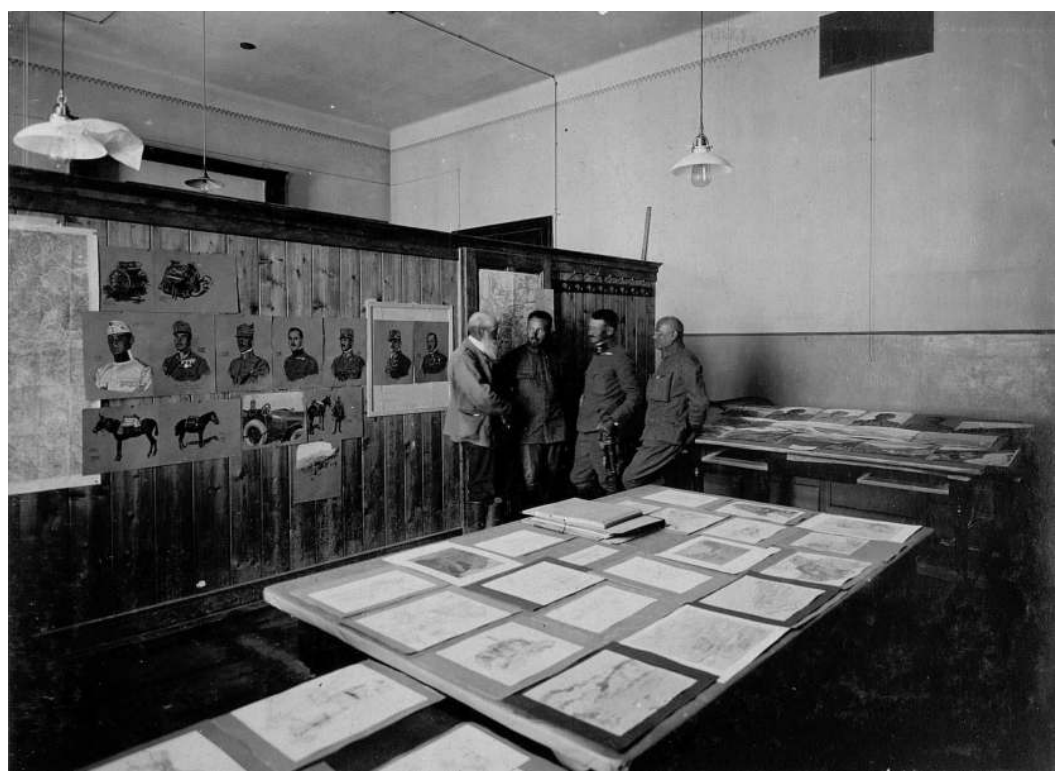

Fig. 3:

Exposition

des peintres

de guerre

(probable-

ment à

Klagenfurt),

organisée

par le K.u.K.

Kriegspresse-

quartier,

Lichtbildstelle

- Wien, 1916,

Österrei-

chische

National-

bibliothek,

WK1/

ALB036/

10091

certains États neutres, comme la Suisse, accueillent ainsi certaines Kriegsbilderausstellungen. C'est notamment le cas de l'exposition itinérante mise sur pied par le puissant Kriegspressequartier de l'Empire autrichien, présentée à Vienne, Budapest et Graz, et qui transite en 1916 par plusieurs grandes villes de Suisse alémanique (Zurich, Berne et Bâle) (fig. 4) ${ }^{(30)}$.

Ces différentes reconfigurations de la «culture de guerre» contribuent à consacrer l'image comme un agent essentiel de la popularisation des savoirs économiques. En Suisse, aucune institution comparable au Deutsches Kriegswirtschaftsmuseum ne voit pourtant le jour durant la guerre ${ }^{(31)}$. Si deux importants centres privés de documentation économique avaient certes été inaugurés à la veille de la Guerre les Archives économiques suisses (Schweizerisches Wirtschaftsarchiv) à Bâle et les Archives pour le commerce et l'industrie (Archiv für Handel und Industrie) à Zurich créées en 1910 - et que les bureaux d'information et de renseignements commerciaux se multiplient à partir de $1914^{(32)}$, ceux-ci n'ont pas, à proprement parler, une vocation pédagogique. Jusqu'en 1917, les expositions nationales et les musées industriels demeurent encore les lieux traditionnels de production et de transmission des savoirs économiques. Inaugurée à Berne en mai 1914, la troisième Exposition nationale persistera du reste dans le souvenir des organisateurs de la Semaine suisse

30 L'exposition est présentée à Bâle, en 1916, au Stadtcasino, puis au Kunstsalon Wolfsberg du Musée national suisse à Zurich. Elle est enfin accueillie par le Kunstsalon Ferdinand Wyss à Berne.

31 Un Musée de la guerre et de la paix (Kriegs-und Friedensmuseum) est bien créé à Lucerne en 1902, mais la Première Guerre mondiale met un terme brutal au projet, si bien qu'il ferme définitivement ses portes en 1919. Voir Walter Troxler, Daniela Walker, Markus Furrer (éd.), Jan Bloch und das internationale Kriegs- und Friedensmuseum in Luzern, Münster, LIT, 2010.

32 Sur ce sujet voir Claire-Lise DeBlü̈, Exposer pour exporter. Culture visuelle et expansion commerciale en Suisse (1908-1939), Neuchâtel, Alphil-Presses universitaires suisses, 2015, p. 159-170. 
comme «un lieu classique de la formation économique des visiteurs" ${ }^{(33)}$. Ceux-ci s'enorgueilliront de poursuivre cette mission éducative selon un schéma profondément renouvelé.

L'« éducation nationale » au service de la «lutte contre la concurrence étrangère » : les débuts de la Semaine suisse (1917)

La Semaine suisse, il est vrai, se distingue du régime traditionnel des expositions, tel qu'il s'était développé au XIX ${ }^{\mathrm{e}}$ siècle. Son originalité réside en effet dans sa capacité à s'affranchir de la halle industrielle et à se déployer sur l'ensemble du territoire national tout en conservant une certaine unité de ton et d'action. Si l'inauguration de la manifestation a lieu en 1917, son origine remonte aux premiers mois de la guerre. L’entrée de la Suisse dans une économie de guerre et les impératifs de la «lutte contre la concurrence étrangère » dictent en effet, dès l'automne 1914, les priorités de l'agenda politique et économique. Plusieurs mesures sont adoptées par la Confédération pour favoriser l'écoulement des «produits nationaux» sur le marché intérieur et encourager la création d'industries nouvelles, dans un contexte où les difficultés d'approvisionnement en matières premières péjorent fortement certaines entreprises. Si quelques secteurs d'exportation, comme l'industrie horlogère ou chimique, parviennent à tirer profit des reconfigurations des relations commerciales, d'autres subissent de plein fouet le retour au protectionnisme et les conséquences des politiques de blocus et de contre-blocus décrétées par les puissances belligérantes. C'est notamment le cas des petites entreprises et des artisans davantage tournés vers le marché intérieur, qui apporteront leur soutien à la Semaine suisse.

Fig. 4: Kriegsbilderausstellung, Werke österreichischer und ungarischer Künstler,

Kunstsalon Wolfsberg,

Musée national suisse Zurich, mai-juin 1916

Graphisme: Carl Hassmann (coll. Bibliothèque nationale suisse)

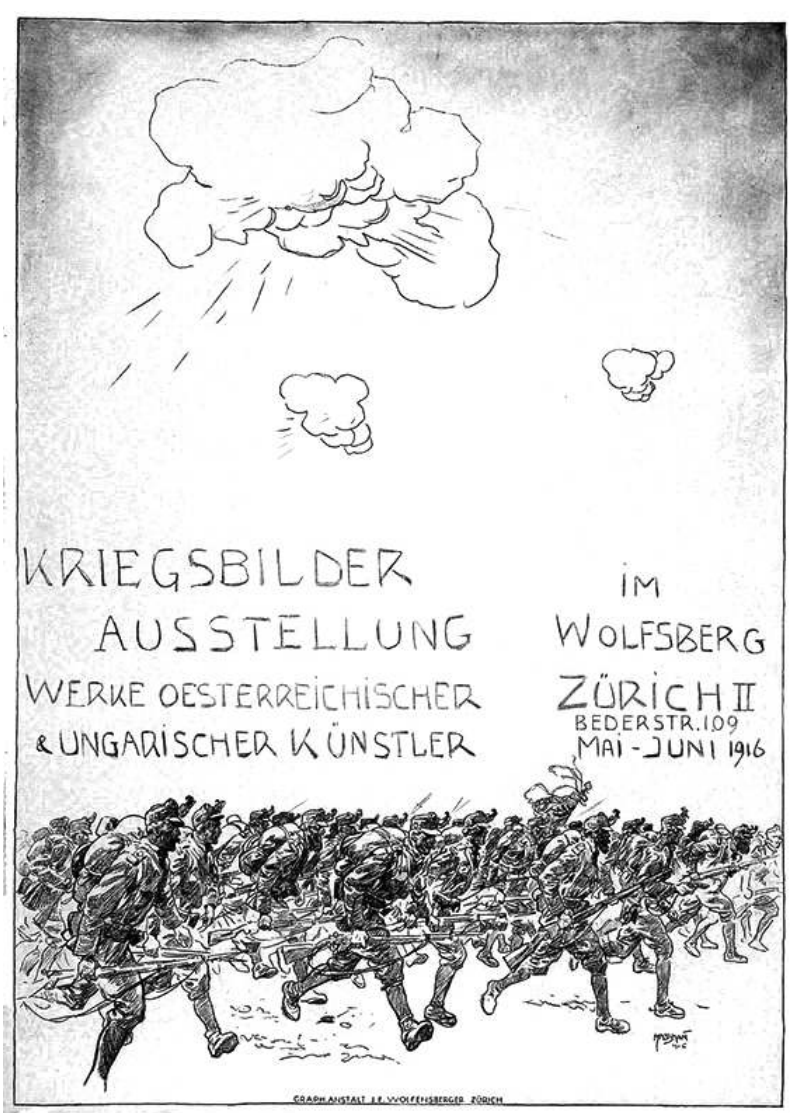

33 «Eine klassische Stätte volkswirtschaftlicher Bildung», in: Conférence du 13.02.1916, tapuscrit, p. 14, CH SWA, Verbandsarchiv Armbrust/Schweizer Woche, PA 486, A 17. 
Dans un climat marqué par de fortes tensions sociales, qui font craindre une sédition des classes populaires appauvries par l'inflation galopante - en quelques mois la valeur réelle des salaires chute de $30 \%$-, les organisations patronales et patriotes se mettent, elles aussi, en ordre de marche. En l'absence d'un service officiel de propagande, certains organismes privés, comme la Nouvelle Société Helvétique (NSH), se substituent à l'État dans la mise en place de campagnes ciblées d'information destinées à contrer l'influence des services étrangers de propagande sur le territoire helvétique. Cette très conservatrice organisation, créée au début de l'année 1914, réunit à ses débuts des intellectuels soucieux d'instaurer un nouvel ordre social pour mettre un terme à l'urbanisation débridée, au «matérialisme» et au «cosmopolitisme niveleur» ${ }^{(34)}$, conçus comme autant de symptômes d'une civilisation en proie à la dégénérescence. Proclamant sa volonté de «renforcer le sentiment national», à l'intérieur des frontières d'abord puis, dès 1916, auprès des «Suisses de l'étranger», la NSH préconise, par la voix de son fondateur, le catholique-conservateur Gonzague de Reynold qui jouit alors d'une influence remarquable auprès des élites intellectuelles et politiques ${ }^{(35)}$, la création de "petits foyers prêts à éclairer l'opinion, à la galvaniser " ${ }^{(36)}$. En homme de lettre et helvétiste averti, de Reynold adhère aux principes d'une "propagande patriotique», dont il contribue dès 1914 à définir les objectifs et les moyens d'action ${ }^{(37)}$. Grâce au soutien décisif de l'Association de la presse suisse et à la collaboration de plus de deux cents titres, la Semaine suisse bénéficie d'une couverture médiatique considérable. À côté des actions menées par les groupes locaux sur le terrain, ce «moyen d'influence indirect " ${ }^{(38)}$ constitue l'une des pierres angulaires du travail d'information et de propagande de la NSH. En 1919, celle-ci étudiera du reste la possibilité de créer son propre service de presse et de propagande afin d'exercer un contrôle plus étroit sur les informations diffusées dans la presse étrangère ${ }^{(39)}$.

Outre les efforts déployés en direction des "Suisses de l'étranger», l'organisation se donne plus particulièrement pour mission de former des groupes de propagande à l'intention de la jeunesse. Un groupe dédié à la question de «l'éducation nationale»

34 Georges de Montenach, «Le Régionalisme», Bulletin de L’Euvre, nº 12 (décembre 1922), p. 4.

35 Voir Aram Mattioli, «Gonzague de Reynold - Vordenker, Propagandist und gescheiterter Chef der 'nationalen Revolution'», in: Aram Mattioli (éd.), Intellektuelle von rechts. Ideologie und Politik in der Schweiz 1918-1939, Zurich, Orell Füssli, 1995, p. 135-156.

36 Lettre de Gonzague de Reynold à H. Tanner (membre du Conseil d'État bâlois), 8 février 1918, Archives littéraires suisses (ALS), Fonds Gonzague de Reynold, B-1.

37 De Reynold prend dès 1914 la tête du Bureau des conférences de l'armée créé par l'État-major helvétique, puis développe un dispositif de «contre-propagande» avec l'appui de la presse régionale et nationale. Voir Alexandre Elsig, «Entre discorde et concorde. La cohésion nationale à l'épreuve des propagandes », in: Roman Rossfeld, Thomas Buomberger, Patrick Kury (éd.), 14/18. La Suisse et la Grande Guerre, Baden, Hier und Jetzt, 2014, p. 85-87.

38 "Wie kann und soll die Neue Helvetische Gesellschaft wirken? Referat, gehalten an der Jahrestagung der Neuen Helvetischen Gesellschaft in Luzern vom 25. September 1915, von F. v. Fischer (Bern)», Nouvelle société helvétique, Bulletin mensuel, supplément (octobre 1915), p. 6.

39 Gérald Arlettaz, "La Nouvelle Société Helvétique et les Suisses à l'étranger (1914-1924). Aspects de la construction d'un nationalisme de type ethnique», Études et Sources, vol. 28 (2002), p. 46. Voir également la lettre de Gonzague de Reynold à Gérard Steck, 11 janvier 1918, ALS, Fonds Gonzague de Reynold, B-1. 
(Untergruppe für nationale Erziehung) ${ }^{(40)}$ est ainsi constitué en 1915, tandis que la commission de propagande de la NSH envisage de généraliser les cours d'instruction civique (Staatsbürgerliche Kurse) ${ }^{(41)}$, dans le but, toujours, de préparer la jeunesse à l'avènement d'un nouvel ordre social. En somme, la NSH prétend non seulement agir sur les domaines de la vie sociale, politique et intellectuelle (de la politique culturelle à la nomination des professeurs d'université) ${ }^{(42)}$, mais également sur celui de l'économie. C'est dans ce sens qu'elle mandate en 1915 l'un de ses groupes régionaux, le groupe de Schaffhouse, une ville de Suisse orientale, pour «s'occuper spécialement des affaires économiques et par conséquent de la pénétration pacifique ${ }^{(43)}$. Après avoir réalisé plusieurs enquêtes sur le sujet, sans grand succès, le groupe de Schaffhouse soumet à l'approbation des autres groupes locaux la création d'une "commission de l'économie nationale" (Volkswirtschaftliche Kommission) à qui il confie l'organisation de la Semaine suisse. «Une entreprise à la fois économique et patriotique est chose neuve ${ }^{(44)}$, assure-t-on alors dans ses rangs, omettant sans doute volontairement d'évoquer d'autres exemples internationaux, à l'instar de la All-British Shopping Week. Lancée en 1910, celle-ci avait connu des débuts difficiles, avant de susciter un nouvel engouement durant la guerre, en particulier dans les colonies britanniques ${ }^{(45)}$. Estimant que la guerre ne peut qu'être favorable au «renforcement du sentiment national» ${ }^{(46)}$, les organisateurs de la Semaine suisse tireront également parti des circonstances singulières pour attirer de nouveaux soutiens. Au moment des premières prises de contact en 1915, ceux-ci estiment ainsi qu'en raison des entraves au commerce extérieur, «le succès de la Semaine Suisse peut être considéré comme assuré si elle est organisée pendant la guerre ${ }^{(47)}$.

Le projet s'était pourtant d'abord heurté à de nombreuses résistances - notamment des milieux de l'exportation, soucieux de ne pas contrarier leurs partenaires commerciaux en souscrivant aux velléités protectionnistes de l'entreprise. Certains acteurs issus de la minorité francophone redoutaient pour leur part l'emprise alémanique sur la manifestation. En 1916, le groupe d'initiative de la Semaine suisse avait enfin traversé une grave crise interne. Au mois de mars, son président Hermann Behrmann, qui dirigeait alors l'Office du tourisme de la Ville de Berne, avait été interpellé par la Police fédérale pour espionnage ${ }^{(48)}$, un scandale pour le moins malvenu de la part

40 «Thesen und Anträge zur Nationalen Erziehung (Im Ausschluss an einen Vortrag der Gruppe Schaffhausen vorgelegt von Dr. Ernst Kelhofer)», Nouvelle société helvétique, Bulletin mensuel, $\mathrm{n}^{\circ} 9$ (juillet 1916), p. 11-12.

41 «An den Vorstand der Neuen Helvetischen Gesellschaft» (s. d.), Staatsarchiv Basel-Stadt (StABS), PA $507, \mathrm{~B} 1$.

42 Document concernant la formation d'un groupe de propagande à l'intention de la jeunesse, 20 février 1918, StABS, PA 507, B1.

43 Arthur Freymond, N.S.H., Lausanne (Tapuscrit), StABS, PA 507, B1.

44 «Proposition du groupe de Schaffhouse à l'ordre du jour du prochain conseil des délégués», Nouvelle société helvétique. Bulletin mensuel, $\mathrm{n}^{\circ} 1$ (septembre 1915), p. 6-7.

45 Frank Trentmann, Free Trade Nation: Commerce, Consumption and Civil Society in Modern Britain, Oxford, Oxford University Press, 2009, p. 229-231.

46 CH SWA, PA 486, A 3. Circulaire du groupe de Schaffhouse non datée (c. 1915).

47 Ibid.

48 «Schweizerwoche. Bericht an die Delegiertenversammlung der N.H.G. vom 3./4. Juni 1916 im Museum in Winterthur», CH SWA, PA 486, A 11. 
d'une organisation qui prétendait lutter contre la «pénétration pacifique». La stupeur provoquée par cette révélation et les inévitables ajustements structurels qui en avaient découlé, avaient entraîné un nouveau retard dans le lancement de la Semaine suisse.

Ce n'est finalement qu'en juin 1917 que l'Association Semaine suisse est créée, plus de deux ans après les débuts de la campagne en faveur de la manifestation. Durant cet intervalle, ses organisateurs étaient parvenus à agréger un nombre remarquable d'acteurs autour de leur projet, bien au-delà de la base historique de la NSH. Face à la durée du conflit, au durcissement des conditions économiques et de la "question sociale", les détracteurs de la Semaine suisse s'étaient progressivement ralliés au projet. Avec le soutien des organisations économiques et patronales, des détaillants, des ligues sociales d'acheteurs, des organisations féminines et des pouvoirs publics, l'Association Semaine suisse avait ainsi relayé le programme de rénovation sociale de la NSH auprès des "cercles commerciaux ou populaires » ${ }^{(49)}$ restés jusqu'alors relativement étrangers aux thèses de l'organisation patriote.

En prétendant éveiller chez le grand public «un sentiment de responsabilité vis-àvis des questions économiques» («volkswirtschaftliches Verantwortungsgefühl»)(50), la NSH fédère ainsi en 1917 un nombre considérable d'acteurs privés et publics autour d'une idée commune: défendre l'indépendance économique du pays et sensibiliser l'opinion aux vertus de la «consommation patriote». Cette faculté à agréger des catégories d'acteurs extrêmement hétérogènes s'explique non seulement par les conditions exceptionnelles entraînées par la guerre, mais également par une volonté de forger un discours «transcendant le langage de l'utilité économique » ${ }^{(51)}$, comme l'a montré Frank Trentmann au sujet des mouvements libéraux britanniques du tournant du $\mathrm{XX}^{\mathrm{e}}$ siècle. À leur instar, les promoteurs de la Semaine suisse contribuent à façonner une figure du consommateur aux contours consensuels, facilement appropriable et propre à mettre d'accord des acteurs aux intérêts parfois diamétralement opposés. Cette figure idéalisée persistera dans le discours de la NSH sous les traits d'un citoyen éduqué aux principes du «patriotisme économique», de la «solidarité» et de l'« effort commun».

\section{Du «musée régional» à l'« exposition nationale décentralisée»: la conquête de l'espace public comme moyen d'édification des masses}

La Semaine suisse prend dès sa première édition la forme d'une «exposition nationale décentralisée», un principe cher aux membres de la NSH, elle-même structurée selon un schéma fédéraliste. Si l'injonction peut paraître paradoxale de la part d'une organisation veillant au renforcement $\mathrm{du}$ «sentiment national», elle se justifie en réalité par une volonté de préserver les particularismes régionaux. Dans un contexte où les tensions entre les différentes régions linguistiques et culturelles de la Suisse sont extrêmement fortes, la prévalence du modèle fédéraliste est essentielle. Chacun des groupes locaux de la NSH doit ainsi surseoir à l'organisation de la manifestation dans sa propre région. Bien qu’inspiré de la All British Week, le principe de la manifestation

49 «Lausanne», Nouvelle société helvétique. Bulletin mensuel, nº 25 (septembre 1917), p. 12 (s. n.).

50 «Bericht über die Durchführung der Schweizerwoche 1918, vom Zentralsekretariat des Verbandes "Schweizerwoche" ", propos rapporté de la Direction de l'enseignement du Canton de Zug, p. 3, StABS, PA 507, C 3.

51 Frank Trentmann, "Political Culture and Political Economy: Interest, Ideology and Free Trade», Review of International Political Economy, 5/2 (été 1998), p. 229. 
ne s'inscrit pas moins dans la filiation du projet de «musées régionaux» développé en 1915 par un autre des membres de la NSH, Georges de Montenach ${ }^{(52)}$. Catholique conservateur, proche des milieux réactionnaires de la Nouvelle droite ${ }^{(53)}$, ce cousin de Gonzague de Reynold en expose longuement les principes dans son essai intitulé Les musées régionaux. Contribution à l'étude du problème de l'éducation nationale, publié sous l'égide de la section fribourgeoise de la Société suisse des traditions populaires. Faisant suite à d'autres essais consacrés à la valeur morale des arts ${ }^{(54)}$, Montenach y développe l'idée selon laquelle les musées régionaux pourraient devenir un «moyen puissant de propagande nationaliste et d'action patriotique ${ }^{(55)}$. L'«édification des masses ouvrières et paysannes à la culture esthétique» est notamment au cœur du projet de l'auteur qui prétend créer dans différentes régions de Suisse «des foyers de beauté sociale ${ }^{(56)}$. Pour ce membre du Heimatschutz, fervent défenseur du patrimoine et de l'art populaire, le fait d'arpenter les rues d'un village pittoresque reconnu pour la qualité de son architecture présente des vertus égales, sinon supérieures, au fait de parcourir les allées d'un musée: "Nous devons, avant tout, agir de telle sorte que nos villages et nos villes restent ou redeviennent des Musées, Musées de plein-air, Musées naturels, Musées vivants", écrit-il ainsi ${ }^{(57)}$. Convaincu des mérites pédagogiques d'un tel projet, Montenach souligne que «les monuments publics et la rue [deviendront] les musées de l'éducation sociale» ${ }^{(58)}$. Les «musées régionaux», tels que Montenach les conçoit en 1915, et la Semaine suisse présentent de nombreux traits communs. Outre l'articulation systématique entre pensée esthétique et patriotisme, on retrouve une même volonté de reconfigurer l'espace publique pour lui assigner une fonction pédagogique.

Doublée d'une dimension commerciale, la Semaine suisse se distingue toutefois des «musées régionaux» et s'inscrit dans un catalogue plus vaste de mesures visant une véritable réforme de l'ordre économique, politique et social, appelé des vœux des milieux néo-patriotes depuis le début du siècle. En recourant aux valeurs et au vocabulaire de la «lutte contre la concurrence étrangère», ceux-ci parviennent à répandre les principes de l'«éducation nationale» avec plus de succès que Montenach lorsqu'il préconisait la création de musées régionaux. Pour la NSH, la Semaine suisse constitue, il est vrai, davantage un «moyen» qu'une fin en $\operatorname{soi}^{(59)}$. L'attrait de la manifestation réside ainsi dans sa

52 Sur ce projet et ses relations au Musée national suisse, voir Chantal LAFontant VALlotton, Entre le musée et le marché. Heinrich Angst: collectionneur, marchand, et premier directeur du Musée National Suisse, Berne, Peter Lang, 2007, p. 46-47.

53 Georges de Montenach, Les Musées régionaux. Contribution à l'étude du problème de l'éducation nationale, Fribourg, Imprimerie de L'œuvre de Saint-Paul, 1915, p. 27.

54 Voir notamment Georges de Montenach, L'art et le peuple, Fribourg, Imprimerie Fragnière Frères, 1903.

55 Catholique militant, député conservateur au Grand conseil puis conseiller d'État fribourgeois, Montenach est une figure centrale de la Nouvelle droite helvétique. Voir Hans Ulrich Jost, Les avant-gardes réactionnaires. La naissance de la nouvelle droite en Suisse, 1890-1914, Lausanne, Éditions d'en bas, 1992, p. 51-52.

56 G. de Montenach, Les Musées régionaux (note 53), p. XVIII.

57 Ibid., p. 18.

58 Ibid., p. 73.

59 «Die Schweizerwoche-Veranstaltung ist nicht Selbstzweck für den Verband, sondern nur Mittel», in: "An die Mitglieder der Neuen Helvetischen Gesellschaft», Derendingen/Schaffhausen, im September 1920, StABS, PA 507, C 3. 
formidable capacité à mobiliser des citoyens établis dans les régions les plus reculées du territoire. L'objectif est de susciter un mouvement populaire en faveur de la «production nationale» qu'une action éducative coordonnée dans les écoles et les foyers, menée par les groupes locaux de la NSH, les différents départements de l'instruction publique, les ligues d'acheteurs et les ligues féminines notamment, vient appuyer.

\section{La pédagogie commerciale au service de l'«éducation nationale»}

Pour les animateurs de la Semaine suisse, qui situent le «renforcement du sentiment national» au cœur de leur programme, la sensibilisation et l'éducation du public ("Aufklärung des Publikums» ${ }^{(60)}$ ) aux sujets économiques constituent un instrument essentiel. Contrairement aux milieux réformistes qui, depuis la fin du XIX ${ }^{\mathrm{e}}$ siècle, s'étaient emparés de l'enseignement de l'économie sociale pour améliorer les conditions d'existence des classes populaires et laborieuses ${ }^{(61)}$, les cours dispensés dans le cadre de la Semaine suisse conservent une visée patriote et morale. Les femmes, les enfants, ainsi que les soldats, sont les principaux destinataires de cette entreprise pédagogique de grande envergure. C'est ainsi «en partant non du haut mais du bas, c'est-à-dire de la multitude des consommateurs eux-mêmes ", que les organisateurs de la Semaine suisse prétendent "fortifier [l']économie nationale» ${ }^{(62)}$. Si à la fin des années 1920 certaines références aux méthodes britanniques du «learning by doing» ou aux théoriciens de l'«éducation nouvelle», comme le pédagogue genevois Édouard Claparède, apparaîtront çà et là ${ }^{(63)}$, ce type d'approches basées notamment sur l'antiautoritarisme marquera finalement assez peu les organisateurs de la Semaine suisse. L’ordre économique nouveau appelé de leurs vœux coïncide en effet davantage avec l'avènement d'un ordre social, nouveau lui aussi, hiérarchiquement structuré et entièrement dévoué à la «communauté nationale».

L'éducation des femmes représente dès 1917 un axe clé de ce programme. En tant que mères et gardiennes du foyer familial d'abord, elles sont considérées comme les principales responsables de la formation de la jeunesse aux valeurs patriotes de l'économie nationale ${ }^{(64)}$. En tant que consommatrices ensuite, elles représentent les plus sûrs agents de la formation des futurs citoyens et citoyennes. L'école et les différents lieux de formation professionnels constituent enfin une plateforme essentielle pour la transmission de ces nouvelles formes de savoirs économiques. Bien introduite auprès des autorités, la NSH trouve d'importants appuis auprès des différents Départements cantonaux de l'instruction publique. Dans le canton alémanique d'Argovie par exemple, les autorités exhortent les écoles à consacrer au minimum une heure d'enseignement à la Semaine

60 Neue Helvetische Gesellschaft. Gruppe Schaffhausen, Schaffhausen, den 11. Juni 1915, NHG Ortsgruppe Basel, StABS, PA 507, C 3.

61 Voir Eduard BALsIGER, Volkswirtschaft und Erziehung. Ein Beitrag zur Frage der Unterstützung des Erziehungswesens durch den Bund, Berne, Bund, 1896.

62 «Semaine suisse», Schaffhouse/Berne, le 25 mai 1917, circulaire, Staatsarchiv Basel-Stadt (StABS), Neue Helvetische Gesellschaft Ortsgruppe Basel, PA 507, C 3.

63 «Éducation nationale», Mitteilungen der Neuen Helvetischen Gesellschaft, n 32 (septembre-octobre 1928), p. 15-16 (s. n.).

64 Voir Hedwig Bleuler-Waser, Die Schweizerfrau als Erzieherin zur Tüchtigkeit und Arbeitsfreude, Zurich, Fretz, 1919. 
suisse. L'instruction civique, l'histoire et la géographie sont considérées comme les matières les plus aptes à inculquer le «sentiment national» ${ }^{(65)}$. Afin de "familiariser les élèves avec la pensée de la Semaine suisse» ${ }^{(66)}$, les professeurs sont encouragés à privilégier les catalogues des expositions nationales successives ou de la Foire suisse d'échantillons dans leurs cours, aux dépens des traditionnels manuels scolaires. Du matériel didactique spécialement mis au point par la «sous-commission de l'éducation nationale» de la NSH est tenu à la disposition des enseignants, mais certaines classes se rendent aussi sur les lieux mêmes de la manifestation, dans les rues commerçantes de leur ville ou de leur village. L'inspection systématique des marchandises exposées dans les magasins est ainsi conçue comme une forme "d'enseignement pratique ${ }^{(67)}$, vouée à agir de manière bien plus persuasive sur les élèves que n'importe quel enseignement théorique. La possibilité d'accueillir de petites expositions de produits suisses au sein même des établissements scolaires est également envisagée: «Regel sollte sein: Wo eine Schule Platz hat, muss auch Raum sein für eine Schaustellung von Schweizerwaren » ${ }^{(68)}$. Si le temps de la Semaine suisse les vitrines se muent pour ainsi dire en école, le phénomène s'inverse lorsque, à leur tour, les établissements scolaires deviennent les vitrines de la production nationale. Dans un cas de figure comme dans l'autre, la Semaine suisse offre aux enseignants la possibilité de dispenser un cours d'économie in situ, selon une démarche empirique dont Anne Rasmussen a souligné combien elle était essentielle pour l'institutionnalisation de nouveaux savoirs ${ }^{(69)}$. L'existence d'une communauté économique nationale, présentée comme un axiome incontestable, se verrait ainsi confirmée par l'expérience de terrain conduite par l'enseignant sur les lieux mêmes de la manifestation. Opposant aux savoirs académiques la force des «cours d'économie pratique» d'un genre nouveau, les animateurs de la Semaine suisse, suivis dans ce sens par ceux de la Foire nationale d'échantillons de Bâle, prétendent non seulement remédier à l'ignorance, mais aussi « rectifier les erreurs des manuels » ${ }^{(70)}$ et imposer leur point de vue dans la définition des ressources pédagogiques jugées les plus légitimes pour l'enseignement.

Pour formuler les principes théoriques et pédagogiques de ce programme, la NSH se tourne enfin vers les écoles de commerce. Le vice-président de la Semaine suisse, Hans Töndury, joue un rôle clé dans le rapprochement de la NSH, dont il est l'un des membres, et des écoles de commerce. Professeur à la Faculté des sciences économiques et sociales de l'Université de Genève dès 1915, Töndury est très impliqué dans la réforme de l'enseignement commercial et consacre de nombreux travaux à cette question $^{(71)}$. Il est non seulement l'auteur d'un essai sur «l'indépendance économique» de

65 Eugène MonoD, «Une base pour l'éducation nationale», Schweizerland, vol. I (1916/1917), p. 97.

66 Je traduis. Bericht über die erste Schweizerwoche, 27. Oktober bis 4. November 1917, Zurich, Druck der Genossenschaft Schweizerische Sonntagsblätter, 1918, p. 8.

67 «Praktischen Anschauungsunterricht», in: R. LüDI, «Die Schule und die Schweizerwoche», Korrespondenzblatt des Bernischen Lehrervereins, 19/4 (1917), p. 77.

68 Ibid., p. 78.

69 Anne Rasmussen, "Sciences et guerres», in: D. Pestre (dir.), Histoire des sciences et des savoirs (note 8), p. 50.

70 «À propos de la Foire», Bulletin de la Foire suisse d’échantillons, n 3 (20 mars 1919), p. 37 (s. n.).

71 Voir par exemple, Hans Töndury, Die Nationalökonomie als Unterrichtsfach, s. 1. (Coire), 1916, ainsi que plusieurs de ses contributions à la Revue suisse des sciences commerciales. 
la Suisse ${ }^{(72)}$, mais il est également à l'origine du plaidoyer vibrant en faveur de la requalification de l'enseignement commercial. Dans la Revue suisse des sciences commerciales, Töndury se prononce en faveur d'une reconnaissance de la "valeur éducative» des branches commerciales ${ }^{(73)}$. Insistant sur les effets de la guerre sur la définition de «l'idéal d'éducation » ${ }^{(74)}$, Töndury défend la thèse selon laquelle les disciplines commerciales poursuivraient, au même titre que les disciplines dites classiques (langues anciennes, littérature, etc.), une «orientation supérieure». C’est ainsi à un enrichissement des contenus classiques de l'enseignement commercial (arithmétique et compatibilité au premier chef) et au «développement des valeurs éducatives des branches commerciales» qu'appelle Töndury. L’organisation, en octobre 1916 à Lausanne, de la Conférence pédagogique de l'Association suisse pour l'enseignement commercial, sur le thème des "voies et moyens propres à développer l'éducation nationale dans les institutions suisses d'enseignement commercial» abonde également dans ce sens: «Aujourd'hui, les questions économiques sont à l'ordre du jour; il faut que la jeunesse apprenne à s'y intéresser aussi » ${ }^{(75)}$, exhorte notamment l'un des orateurs de la Conférence. La Semaine suisse offre ainsi aux acteurs de la discipline une occasion unique d'apporter la preuve de l'utilité de l'enseignement commercial. Durant la guerre, la volonté de populariser les savoirs économiques est ainsi étroitement liée au processus de légitimation de ces mêmes savoirs au sein des sciences sociales.

\section{Science de l'étalage et ordre visuel: entre exaltation du «sentiment national» et quête de l'objectivité}

À côté de l'éducation des consommateurs proprement dite, la formation des commerçants représente une part non négligeable du programme éducatif porté par la NSH. «Pour les exposants, la Semaine suisse constitue un lieu de formation continue», relève ainsi le rapport publié au terme de la première édition de la Semaine suisse ${ }^{(76)}$. En tant qu'intermédiaires entre les producteurs et les consommateurs, les commerçants jouent en effet un rôle déterminant dans la diffusion de la doxa patriote de la «lutte contre la concurrence étrangère». Un panneau-réclame aux couleurs de la Semaine suisse certifiant l'origine des produits exposés doit être disposé bien en vue dans la vitrine (fig. 5). Les marchandises étrangères sont prohibées et traquées par les représentants de la Semaine suisse qui n'hésitent pas à mettre à l'index les détaillants en infraction avec le règlement de la manifestation. Cette prescription, tournée en dérision par la revue satirique Nebelspalter qui met en scène un commerçant pressé

72 Hans Töndury, Wirtschaftliche Unabhängigkeit?, Vortrag geh. den 15. Jan. 1915 in der Gruppe St. Gallen der Neuen Helvet. Gesellschaft, Zurich, Rascher, 1915.

73 Hans TöNdury, «La valeur éducative des branches commerciales », Revue suisse des sciences commerciales, $\mathrm{n}^{\circ} 2$ (mars 1916), p. 49.

74 Ibid., p. 54.

75 Adolphe BlASER, "Voies et moyens propres à développer l'éducation nationale dans les institutions suisses d'enseignement commercial», Revue suisse des sciences commerciales, $\mathrm{n}^{\circ} 11$ (novembre 1916), p. 270.

76 «Die Schweizerwoche ist eine gute Fortbildungsschule für die Aussteller [...]», propos rapportés dans Bericht über die erste Schweizerwoche, 27. Oktober bis 4. November 1917, Zurich, Druck der Genossenschaft Schweizerische Sonntagsblätter, 1918, p. 20. 

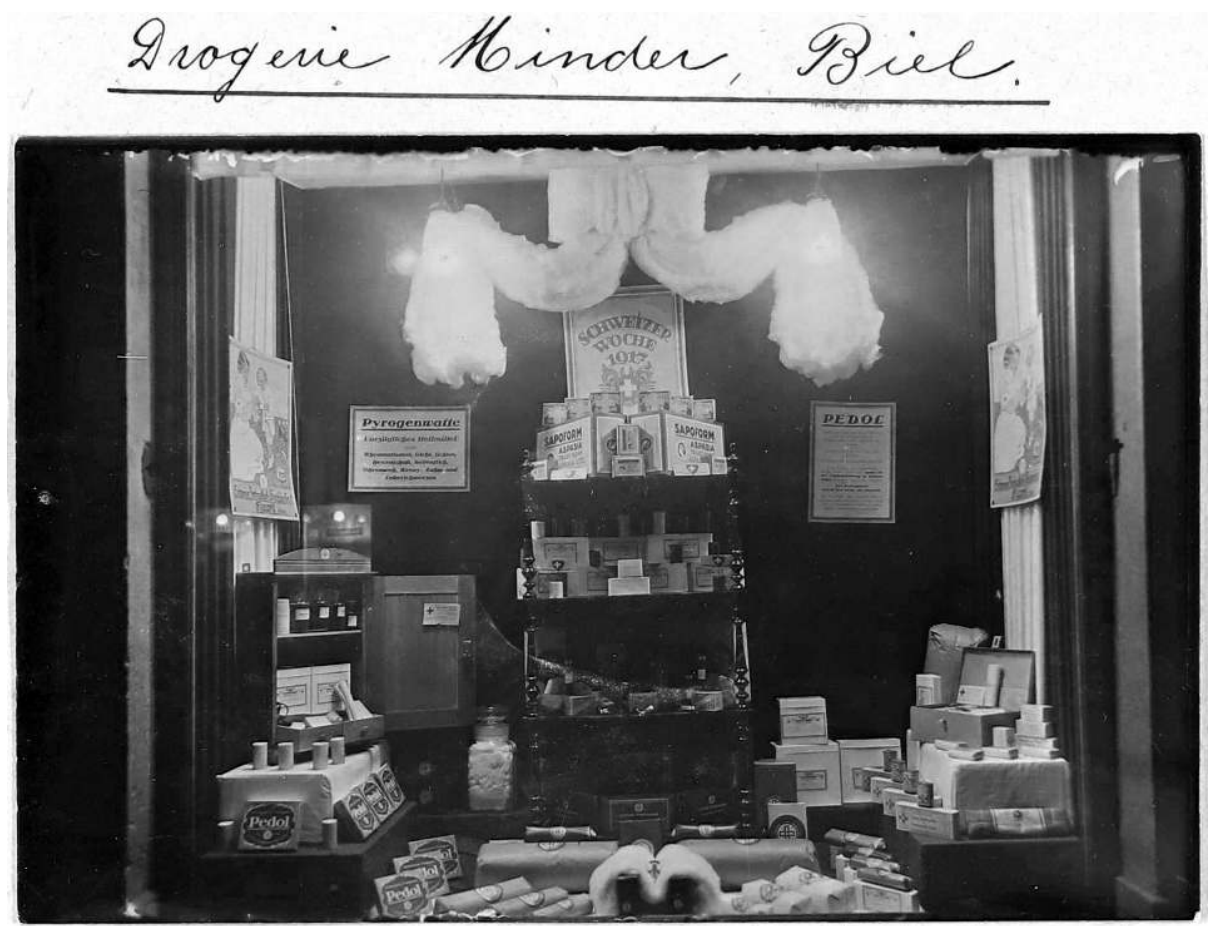

Fig. 5: Vitrine de la Droguerie Minder à Bienne durant la Semaine suisse 1917, CH SWA PA 486 B 91

d'exposer à nouveau des produits importés, et jetant à terre les décorations patriotes utilisées durant la Semaine suisse, ne fait, il est vrai, pas toujours l'affaire des détaillants (fig. 6). C'est à eux pourtant qu'incombe la responsabilité d'attirer le consommateur dans leur magasin, en déclarant leur attachement aux produits suisses et en portant un soin particulier à leurs aménagements. Ainsi que le proclame le slogan officiel de la manifestation, il s'agit de faire «honneur au travail national».

Pour appuyer les commerçants dans cette tâche, plusieurs manuels sont édités sous l'égide de la Semaine suisse. Les prescriptions en matière de décoration forment la matière principale d'un court ouvrage paru à l'occasion de sa première édition: Die Ausstattung der Schaufenster ${ }^{(77)}$. Rédigé par un commerçant originaire de Soleure, Johann Kälin, ce texte situé à mi-chemin entre le manuel professionnel et l'essai peut être lu comme un catalogue des dispositions élémentaires à observer en matière de décoration de vitrine. Pour l'auteur de ce bref opus, la vitrine ne saurait être réduite à un simple étal de marchandises. Elle constitue au contraire un puissant véhicule des valeurs patriotes portées par la Semaine suisse. Le décorateur ou l'étalagiste sont ainsi présentés comme les agents d'une mutation profonde des habitudes de consommation et des représentations collectives de l'économie.

77 Johann KäLIN, Über Ausstattung der Schaufenster. Einige Winke und Anleitungen, Olten, Oltner Druck- \& Verlagsanstalt, 1917. 


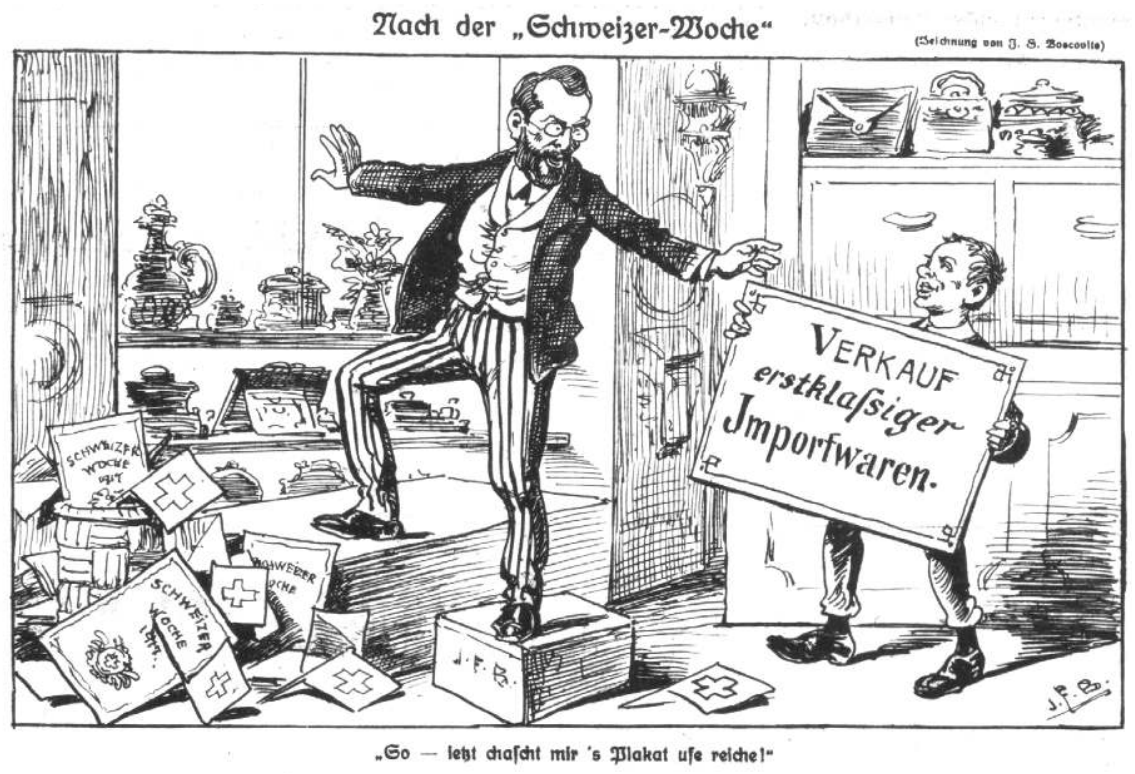

Fig. 6: Après la Semaine suisse. «- Voilà, maintenant tu peux me passer l'affiche!» (sur l'affiche: "Vente de produits importés de première classe»)

Caricature parue dans Nebelspalter, 43/45 (1917), (s. p.)

L'aménagement de la vitrine repose sur un principe de parcimonie qui répond aux difficultés conjoncturelles de la période. L'objectif est non seulement de rompre avec le principe de profusion qui avait dominé les expositions de la deuxième moitié du $\mathrm{XIX}^{\mathrm{e}}$ siècle, mais aussi de limiter les frais entraînés par de tels aménagements: «In der Beschränkung zeigt sich erst der Meister », indique ainsi Kälin ${ }^{(78)}$. Arrangées avec soin et méthode, les vitrines doivent offrir le visage d'un étalage systématiquement ordonnancé. La feuille de route de la «décoration de vitrine» est inspirée des principes hygiénistes enseignés depuis la fin du XIX ${ }^{\mathrm{e}}$ siècle aux vendeuses et aux «répartitrices» des coopératives de consommation (fig. 7) ${ }^{(79)}$. La lumière doit être abondante, la marchandise, soigneusement étalée et régulièrement renouvelée. La publication se distingue toutefois d'autres manuels rédigés à l'intention des détaillants. Il s'agit non seulement de définir la meilleure manière d'écouler des produits, mais aussi de convaincre le public des vertus de la consommation patriote. Pour déployer entièrement ses effets, l'aménagement des vitrines doit ainsi faire appel à la subjectivité des consommateurs.

Le principe même d'une «décoration patriotique» (patriotische Dekoration) est longuement développé. Dans le cas où il ne pourrait s'offrir les services d'un décorateur, le commerçant doit veiller à respecter un certain nombre de dispositions, en particulier en ce qui concerne les usages « patriotiques» de la couleur. Le sujet est loin d'être secondaire puisque l'intégralité d'un chapitre est consacrée à cette question. Selon les prescriptions d'usage

79 Émile Schwarz, Guide pratique pour le service du magasin coopératif, Bâle, Union suisse des Sociétés de consommation, 1912 (s. p.). 
Fig. 7: «Vues de dépôts de coopératives dont le bon ordre peut servir de modèle"

Émile Schwarz, Guide pratique pour le service du magasin coopératif, Bâle, Union suisse des sociétés de consommation, 1912, [s. p.]

décrites par Kälin, une vitrine ornée de guirlandes ou de morceaux d'étoffe aux tonalités proches du drapeau national (le rouge et le blanc) agirait sur «les yeux et le cœur ${ }^{\left({ }^{(80)}\right.}$ bien plus sûrement qu'un aménagement classique. Donnant libre cours à sa propre interprétation de la psychologie des couleurs, cette science si répandue à la fin du XIX ${ }^{e}$ siècle, Kälin appelle toutefois à une certaine retenue. Un recours par trop appuyé au rouge et au blanc pourrait durement «frapper l'esprit » ${ }^{(81)}$, estime-til. Sous les apparences d'un discours scienti:: Vues de dépôts de coopératives :: dont le bon ordre peut servir de modèle

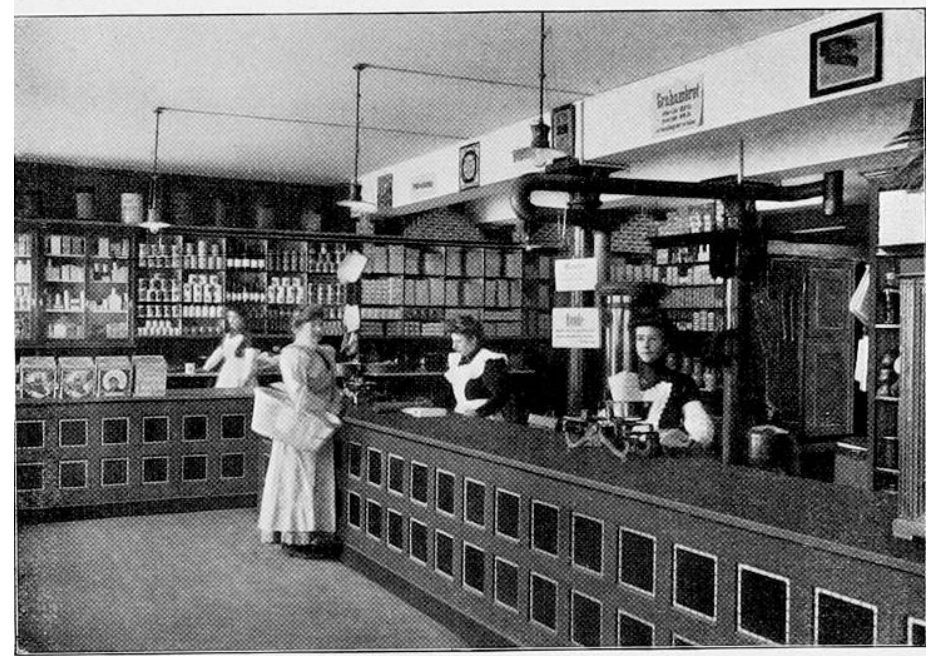

Société générale de consommation à Bâle

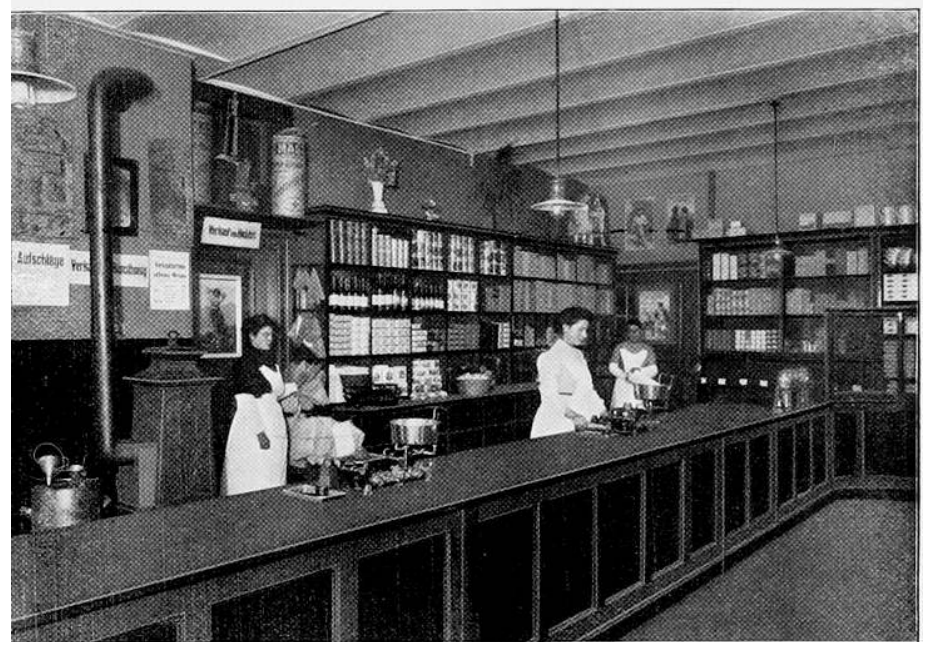
fique établissant une claire distinction entre les différentes fonctions des couleurs, comme certains statisticiens s'y étaient également employés avant lui, Kälin développe une approche prétendument empirique de l'«économie nationale » qui fait davantage appel aux émotions de la «grande armée des consommateurs» ${ }^{(82)}$ qu'à leur jugement exercé.

80 Je traduis. J. KäLIN, Über Ausstattung der Schaufenster (note 77), p. 13.

81 Ibid.

82 Semaine suisse (note 3), p. 13. 
Contrairement à la Semaine suisse, les nombreuses foires d'échantillons créées dans le sillage de la Grande Guerre ont pour ambition de re-professionnaliser le champ des expositions. Parmi la presse et dans les rangs mêmes des exposants, il était d'usage, depuis la fin du XIX ${ }^{\text {e }}$ siècle, de déplorer le glissement opéré par les expositions vers le divertissement de masse. Devenues, avec l'éclatement de la guerre, des lieux incontournables du négoce international, les foires d'échantillons s'adressent essentiellement à un public informé (commerçants, négociants et acheteurs en gros), renouvelant par conséquent les pratiques de l'exposition ${ }^{(83)}$. À Bâle, où la Foire nationale suisse d'échantillons est inaugurée en 1917, le vocabulaire utilisé pour encadrer les pratiques de l'étalagisme s'écarte ainsi des usages en vigueur au sein de la Semaine suisse. À l'idée d'une décoration "patriote» faisant appel aux sens et aux sentiments des consommateurs s'oppose celle d'une décoration «objective», établie selon les principes de la «science publicitaire» naissante. Le devoir d'objectivité dans l'aménagement des stands est de "première nécessité » estime-t-on en effet ${ }^{(84)}$. La qualité et l'unité formelles des aménagements sont ainsi conçues comme les gages d'une organisation moderne et rationnelle de l'économie. «L'observation objective » à laquelle inviteraient les aménagements réalisés «sans aucune recherche de l'effet " ${ }^{\left({ }^{85}\right)}$ constitue une expression majeure du mouvement de professionnalisation qui s'est emparé, depuis les débuts de la guerre, des foires commerciales. "Ni mesquin, ni somptueux, mais objectif ${ }^{(86)}$, l'aménagement des stands prétend allier économie de moyens et rigueur méthodologique. Ce mode singulier d'adresse, destiné à "l'acheteur pressé» ${ }^{(87)} \mathrm{de}$ passage à Bâle, permet en outre de développer une forme de discours prétendument scientifique, à une période où les excès de la propagande sont couramment dénoncés. Dans ce sens, l'«objectivité» des aménagements devient un gage d'authenticité, renouant avec la croyance en la transparence de la représentation, si prégnante dans le champ des sciences au XIX ${ }^{\mathrm{e}}$ siècle. L'idée même d'établir des «modèles» s'apparente à une démarche de type empirique inspirée, elle aussi, par la pratique des sciences. Dès ses premières livraisons, à partir du mois de janvier 1916, le bulletin de la foire publie plusieurs vues de "stands modèles", qui «renseigneront les amateurs, mieux que de longues explications, sur l'importance de la technique d'exposition appropriée aux exigences actuelles» ${ }^{(88)}$. Les vues des «stands-types» publiées dans le périodique sont accompagnées de longues légendes explicatives, indispensables à l'interprétation des images (fig. 8). Même reproduites en grand format, elles restent peu lisibles et montrent que sans la présence d'un appareil didactique, la pédagogie par l'image rencontre de nombreuses limites.

83 Voir Claire-Lise Deblü̈, «De l'exposition universelle à la foire nationale. Éléments pour une histoire élargie des expositions durant la Première Guerre mondiale», Relations internationales, 4/164 (2015), p. 59-74.

84 Alfred Ditisheim, «Étude sur la manière de présenter les modèles à la Foire suisse d'échantillons », $L a$ Foire suisse d'échantillons, $\mathrm{n}^{\circ} 1$ (15 novembre 1916), p. 72.

85 Ibid., p. 71.

86 «Musterstand - Modèle de stand», La Foire suisse d'échantillons, n 7 (15 février 1917), p. 221 (s. n.).

87 A. Ditisheim, «Étude sur la manière de présenter les modèles» (note 84), p. 72.

88 Ibid. 
Dans un climat économique pour le moins incertain, la production d'un discours objectivant, aux prétentions scientifiques, répond à la nécessité de légitimer l'entreprise de la foire auprès des acheteurs et d'éventuels exposants. L'année suivante, la direction de la Foire développera d'ailleurs une offre de cours de formation et de conférences destinée aux exposants. À la demande de la direction de la Foire, l'École des Arts et Métiers de Bâle mettra même sur pied un cours de décoration de vitrine ${ }^{(89)}$, qui donnera lieu, à la clôture de la Foire, à une exposition de réclame artistique au

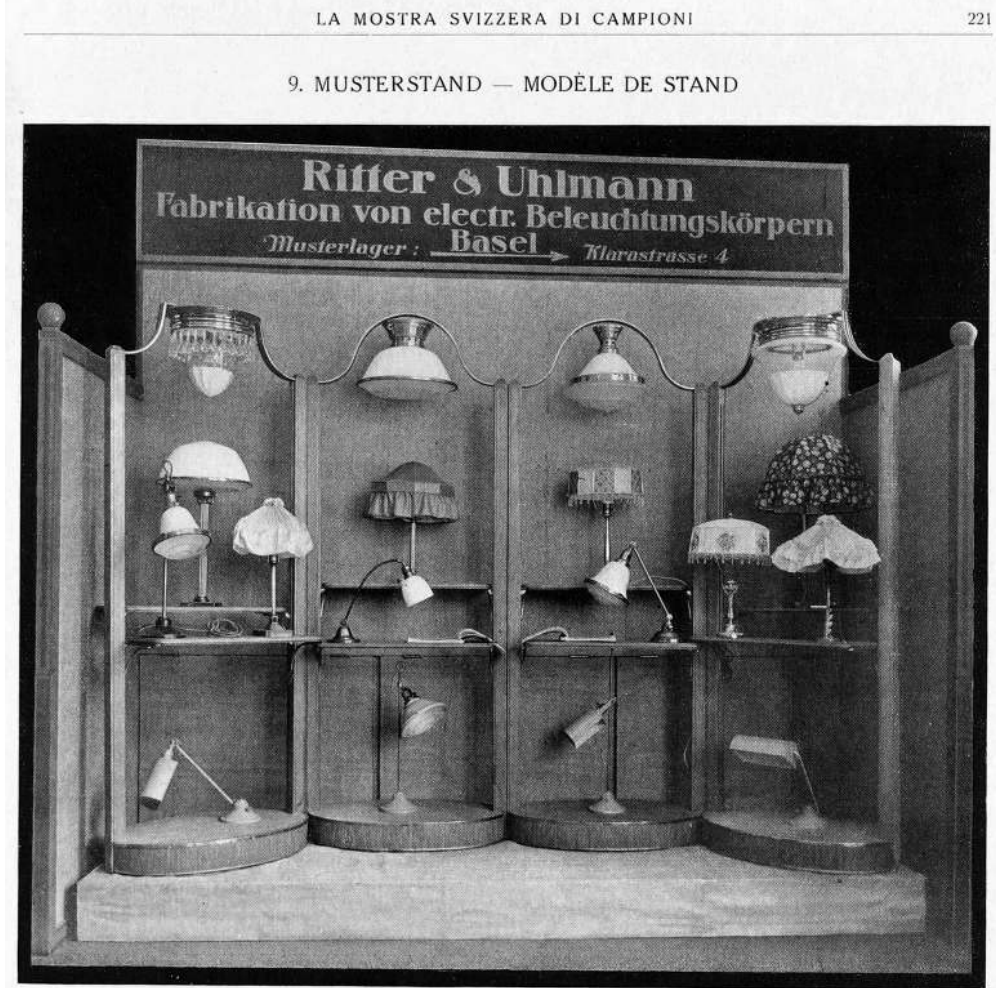

\footnotetext{
Er soll zeigen, wie man sich auch mit altem Ausstellungsgerăt behelfen kann, wenn dasselbe nicht bloss spielerischer Art oder
zum Prunken geschaffen ist, sondern sich als rein sachlich erweist. Gerade bei einem Stand, wo Hăngelampen verschiedener Art und Tischlampen zu verschiedener Verwendung kommen, wird es unmöglich sein, sich mit einem Tisch zu begnügen. Damit schliessen wir die Serie der Musterstande und werden in den nächsten Heften ausstellungstechnische Mitteilungen anderer Art bringen.

Ce stand mesure $3 \mathrm{~m}^{2}$ de surface-plancher; location frs. 230 .

L'illustration ci-dessus a pour but de démontrer comment il est possible d'utiliser un ancien agencement d'exposition à condition qu'il ne soit ni mesquin ni somptueux mais exclusivement objectif. Il est naturel qu'une stalle présentant des lustres à suspension, des plafonniers, des lampes murales, des candélabres, etc. ne saurait s'accommoder d'un seul support en forme de table.

Cette description clôt la série des stands-modéles; le prochain fascicule donnera dautres renscignements sur la technique des expositions.
}

21

a 0 ㅁ

Fig. 8: «Musterstand - Modèle de stand»

La Foire suisse d'échantillons, $\mathrm{n}^{\circ} 7$ (15 février 1917), p. 221

89 «Kurs für Schaufenster-Dekoration and der Allg. Gewerbeschule Basel», 20.9.1917, StABS, Protokolle der Kommissionssitzungen der Allgemeine Gewerbeschule, T 33.4. 
Gewerbemuseum $^{(90)}$. C'est donc sur l'impulsion décisive de la Foire de Bâle que se structurera progressivement l'activité des décorateurs-étalagistes dans la région bâloise et que l'on assistera à l'institutionnalisation d'une véritable «culture de la vitrine» durant l'entre-deux-guerres ${ }^{(91)}$.

«Élargir l'affectation de la vitrine» pour «informer le public et influencer son opinion ${ }^{(92)}$, tel est en somme l'objectif de la Semaine suisse. En désignant la rue comme plateforme privilégiée de la transmission des savoirs économiques, la Semaine suisse mobilise un répertoire pédagogique largement inédit et s'impose dès 1917 comme un acteur clé de la formation commerciale et patriote. Dans l'immédiat après-guerre, les animateurs de la Semaine suisse diversifient encore leurs moyens d'action en multipliant les conférences accompagnées de projections lumineuses et les présentations cinématographiques. Conçues comme un moyen de «compléter» et de «renfor[cer] l'effet des exhibitions dans les vitrines " ${ }^{(93)}$, ces différentes initiatives contribuent à élargir la zone d'influence de la Semaine suisse qui ambitionne, par ce moyen, d'atteindre les régions les plus reculées du territoire. Confrontés à un public peu familiarisé avec les questions économiques, les organisateurs de la Semaine suisse cherchent en effet dès 1917 à forger un contre-discours au diagnostique des milieux réformistes qui dénoncent à la même période les ravages du renchérissement et la persistance des inégalités sociales, en faisant un pari singulier: celui d'aller directement au contact des consommateurs. "Tous les habitants du pays seront involontairement les visiteurs de cette exposition nationale décentralisée et achèteront naturellement des produits indigènes " ${ }^{(94)}$, indiquent ainsi les promoteurs de la manifestation lors de sa première édition.

Loin de l'idéal réformiste de la démocratisation des savoirs ${ }^{(95)}$, le programme éducatif formulé par la NSH constitue un instrument remarquable pour façonner de nouvelles représentations collectives de l'économie et redéfinir sa fonction sociale au sein de la «communauté nationale». Devenue un support privilégié de la circulation des savoirs économiques, la vitrine contribue à répandre et à naturaliser certaines notions comme celles d'«économie nationale» ou d'«éducation nationale». À rebours

90 Protokolle der Kommissionssitzungen der Allgemeine Gewerbeschule, 20.9.1917, StABS, T 33.4.

91 Voir les contributions réunies dans Ruth K. Scheel (éd.), Schaufensterkultur. Inszenierte Warenwelt in Basel, Bâle, Merian, 2013.

92 «Hier bietet die Schweizerwoche dem in seinem Fache umfassend gebildeten Teilnehmer Gelegenheit, die Zweckbestimmung des Schaufensters zu erweitern und belehrend und bildend auf das Publikum einzuwirken», in: "Bericht über die Durchführung der Schweizerwoche 1918, vom Zentralsekretariat des Verbandes Schweizerwoche», p. 11, StABS, Fonds Neue Helvetische Gesellschaft, Ortsgruppe Basel, PA 507, C 3.

93 Semaine suisse. Association nationale en faveur du développement de l'intérêt public pour les questions vitales de l'économie suisse. IVe Rapport annuel 1920-1921, Neuchâtel, Imprimerie Delachaux et Niestlé S.A., 1921, p. 15.

94 Circulaire du groupe de Schaffhouse, janvier 1917, CH SWA, PA 486, A 14.

95 Voir Julie K. Brown, «Making 'Social Facts' Visible in the Early Progressive Era: The Harvard Social Museum and Its Counterparts", in: Deborah Martin KaO, Michelle LAmunière (éd.), Instituting Reform: the Social Museum of Harvard University, 1903-1931, Cambridge, Harvard Art Museums, 2012, p. 93-109. 
de l'abstraction statistique, c'est en disposant leurs produits de manière à "répandre dans le peuple suisse une compréhension plus approfondie des faits économiques» que les détaillants sont invités à «éveiller le sentiment de la solidarité économique» auprès de leurs clients ${ }^{(96)}$. L'abandon de la statistique, d'ordinaire si prisée dans les expositions, cède ainsi la place à des aménagements plus classiques, inspirés des traités d'étalagisme. La "valeur vulgarisatrice» ${ }^{(97)}$ attribuée aux étalages et la confiance placée dans les décorateurs, dont la faculté à livrer une « démonstration graphique " (98) des vertus du patriotisme économique n'est jamais démentie, soulignent la dimension empirique du programme éducatif de la NSH. C'est ainsi qu'en l'espace de quelques années, certaines notions appartenant au répertoire de l'«économie nationale», soigneusement sélectionnées par les organisateurs de la Semaine suisse, ont non seulement investi la sphère du quotidien, mais se sont véritablement déployées dans l'espace publique, bien au-delà des lieux traditionnellement dédiés à l'apprentissage (écoles, universités, musées, etc.). Bien avant que la presse illustrée ou publicitaire ne s'empare de ce type de sujets, la Semaine suisse présente ainsi un cas emblématique des reconfigurations qui, au sortir de la guerre, affecteront la formation et la circulation des savoirs économiques. Leur passage de la sphère académique à la culture de masse s'incarnera aussi bien dans les discours publicitaires que dans les campagnes de propagande orchestrées par le gouvernement helvétique durant la Deuxième Guerre mondiale et qui situeront la "popularisation des principes de l'économie de guerre ${ }^{(99)}$ au cour de leur programme.

\section{Résumé}

L'année 1917 se signale par l'organisation d'une campagne de propagande patriote et économique de grande envergure prenant pour cible la "concurrence étrangère». Directement issue du régime de l'économie de guerre, la Semaine suisse (Schweizer Woche) s'invite dans les écoles, auprès des soldats mobilisés et dans les foyers. Mais elle se déploie surtout dans les vitrines des détaillants, dont les aménagements célèbrent les produits de fabrication helvétique. Dans un contexte marqué par les difficultés d'approvisionnement et l'inflation persistante, la manifestation a non seulement pour vocation d'éduquer les consommateurs, mais aussi d'infléchir les représentations de l'économie dans l'espace public. Resituée dans une perspective transnationale, cette étude de cas s'interroge sur la popularisation des savoirs économiques en temps de guerre. Elle montre notamment qu'au terme du long XIX siècle qui fut le témoin de l'institutionnalisation et de la professionnalisation de l'économie, la rue s'imposa comme un lieu privilégié de diffusion et d'acquisition des connaissances durant la Première Guerre mondiale.

96 Hans Töndury, «L'économie nationale», Journal de Genève, nº 283 (15 octobre 1928), p. 7.

97 H. G., «Le rôle économique de la Semaine suisse», Le Commerçant, n 4 (septembre 1918), p. 106.

98 Ibid.

99 Voir Hans Schaffner, Panorama de l'économie de guerre, Berne, Service d'information de la Centrale fédérale de l'économie de guerre, 1943, p. 3. 


\section{Zusammenfassung}

Im Rahmen der Schweizerischen Kriegswirtschaft wird 1917 eine grossangelegte Propagandaaktion organisiert, die sich gegen die sogenannte „wirtschaftliche Überfremdung"wendet. Die Schweizer Woche richtet sich an mobilisierte Soldaten und lädt sich in Schulen und Privathaushalte ein. Am Sichtbarsten wird sie in den Schaufenstern des Detailhandels, dessen Auslagen helvetische Produkte anpreist. Indem sie ein „volkswirtschaftliches Verantwortungsgefühl“ zu wecken sucht, reagiert die Schweizer Woche insbesondere auf Versorgungsschwierigkeiten und die Inflation, welche die Bevölkerung während des Krieges in wirtschaftliche Not bringen. Während sie einerseits KonsumentInnen über richtiges Konsumverhalten aufklärt, bedingt die Propagandaaktion auch eine zunehmende Darstellung von ökonomischem Wissen im öfentlichen Raum.

Dieser Aufsatz diskutiert anhand des Beispiels der Schweizer Woche die Entstehung und Verbreitung von ökonomischem Wissen um 1917 aus einer transnationalen Perspektive. Er zeigt insbesondere, dass nach einer ersten Phase der Institutionalisierung und Professionalisierung im 19. Jahrhundert die Volkswirtschaft sich anfangs des 20. Jahrhunderts ein neues Publikum erschliesst und neue, öffentliche Räume erobert. Diese neuen Orte der Wissensverbreitung umfassen auch die Ladenstrasse, welche seit dem Ersten Weltkrieg als privilegierter Ort für die Popularisierung von Wissen und den Wissenserwerb gilt. 